\title{
Imatinib-based therapy in adult Philadelphia chromosome-positive acute lymphoblastic leukemia: A case report and literature review
}

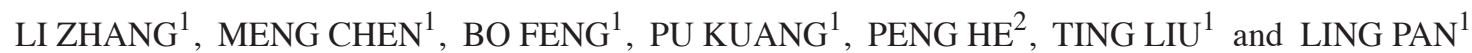 \\ ${ }^{1}$ Department of Hematology, West China Hospital, Sichuan University, Chengdu, Sichuan 610041; \\ ${ }^{2}$ Third Division of Internal Medicine, District People's Hospital, Qionglai Medical Center Hospital, Qionglai, \\ Sichuan 611530, P.R. China
}

Received September 9, 2014; Accepted June 11, 2015

DOI: $10.3892 / 01.2015 .3539$

\begin{abstract}
Acute lymphoblastic leukemia (ALL) has a rapid onset and rarely occurs with exclusive prodrome of general osteoporosis and vertebral compression fractures. However, Philadelphia chromosome-positive $\left(\mathrm{Ph}^{+}\right)$ALL has a poor prognosis, even when patients are treated with intensive chemotherapy, and the first-line effective treatment requires further elucidation. The present study focused on a 56-year-old Chinese male patient who initially presented with spontaneous bone fractures and was ultimately diagnosed as $\mathrm{Ph}^{+} \mathrm{ALL}$ after 6 months, which required to preliminarily exclude a working diagnosis of myeloma. Apart from intensive chemotherapy, the patient successfully completed an imatinib-based regimen and achieved complete remission (CR) 2 weeks later. Subsequently, the patient was subjected to consolidation treatment using the same imatinib regimen combined with interferon- $\alpha 2 b$ for 9 courses. In November 2013, the patient had achieved persistent hematological and molecular genetic normality for $\sim 16$ months after the initial CR. In conclusion, $\mathrm{Ph}^{+} \mathrm{ALL}$ must be considered in the differential diagnosis of adults experiencing unexplained bone disease.
\end{abstract}

\section{Introduction}

Acute lymphoblastic leukemia (ALL) is a neoplasm of precursor cells committed to the B-cell and T-cell lineages involving the bone marrow and blood (1). ALL occasionally presents with primary involvement of nodal sites. Osteoporosis and spontaneous vertebral compression fractures as presentations of ALL have been described mainly in pediatric patients, but rarely in adult patients with ALL $(2,3)$. Philadelphia

Correspondence to: Professor Ling Pan, Department of Hematology, West China Hospital, Sichuan University, 37 Guoxue Lane, Wuhou, Chengdu, Sichuan 610041, P.R. China E-mail: hematologyhuaxi@163.com

Key words: osteoporosis, fracture, acute lymphoblastic leukemia, imatinib chromosome-positive $\left(\mathrm{Ph}^{+}\right)$ALL accounts for $25-30 \%$ of adult ALL and its incidence increases with age in adults $>40$ years old. Prior to the era of tyrosine kinase inhibitor (TKI) treatment, $\mathrm{Ph}^{+} \mathrm{ALL}$ was considered to be a high-risk subgroup among the ALL population. Current intensive chemotherapy regimes have improved the complete remission (CR) rate, however, long-term overall survival (OS) has not improved. In the TKI era, dramatic changes in therapeutic efficacy have occurred, with markedly improved molecular CR and improved OS rates (4). These changes raise questions as to whether the conventional intensive combined chemotherapy treatment regime should remain the front-line treatment strategy for patients with $\mathrm{Ph}^{+}$ALL.

The present study reported the case of a $\mathrm{Ph}^{+}$ALL patient who was treated with imatinib-based individual therapy, which included conventional standard-dose chemotherapy, rather than intensive combined regimens. Imatinib is a first generation TKI, which specifically targets the adenosine triphosphate binding site of the BCR/ABL kinase domain. The current case was characterized by initial presentation of general osteoporosis and vertebral compression fracture.

\section{Case report}

A 56-year-old previously healthy male was originally referred to the West China Hospital (Sichuan University, Chengdu) in June 2012. The patient presented with a 6-month history of progressive lower back and bilateral rib pain. Initially, the patient experienced sudden onset of sharp lower back pain, and a spine X-ray in the local community clinic indicated slipped disc at L2-3, L3-4 and L4-5 levels with a normal complete blood count and white cell differentiation. Acute pain was usually resolved spontaneously, but pain recurrence was frequent. After 3 months, the perceived intensity of pain became more aggressive, followed by emergence of fatigue and dizziness. A repeated complete blood count indicated a hemoglobin level of $6.5 \mathrm{~g} / \mathrm{dl}$ [normal range (NR), 13.0-17.5 g/dl] and platelet count of $15,000 / \mu 1$. A computed tomography (CT) scan revealed large absorption and compression fracture of T8 and L3. Red blood cell transfusion was performed in the local clinic prior to admission to the West China Hospital. Upon 
A

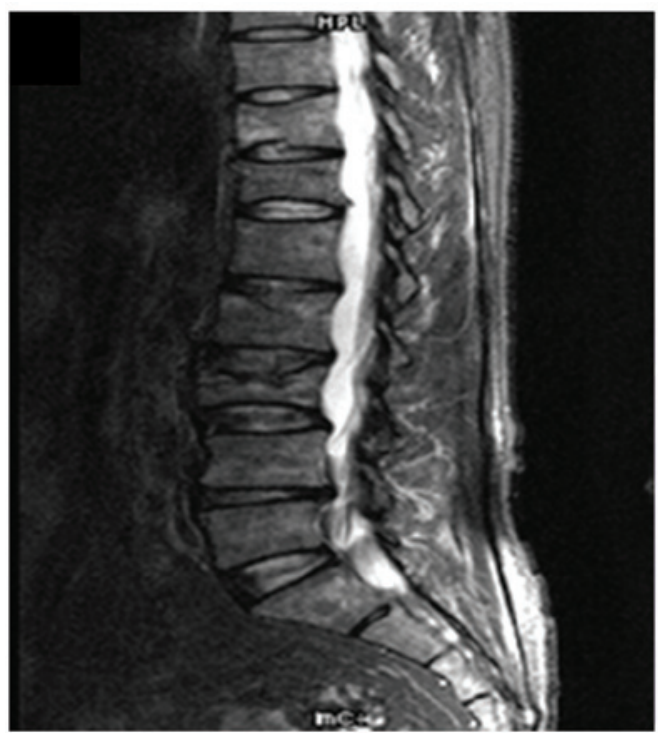

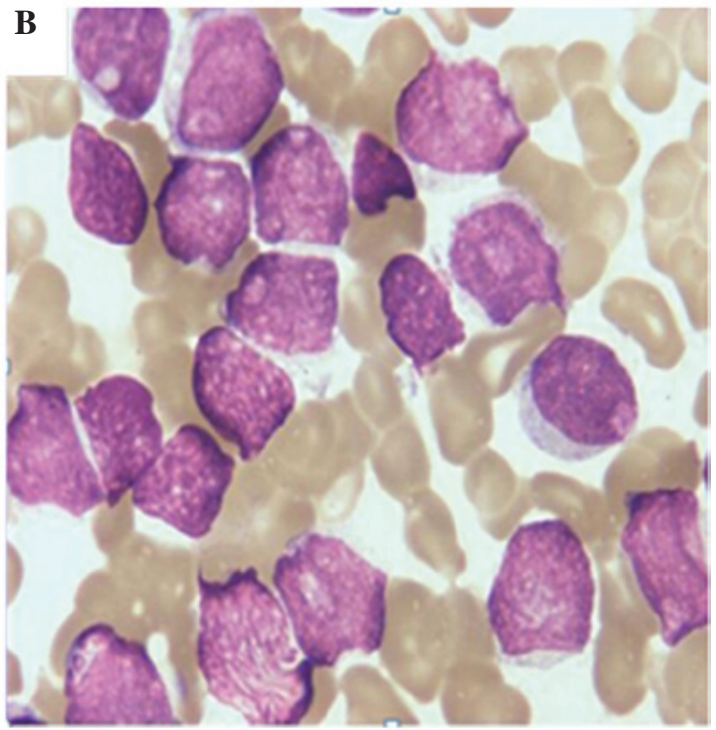

Figure 1. (A) Magnetic resonance imaging revealed impaired physiological curve of thoracolumbar spine, clusters of iso/hypo mixed signals and pathological fracture in T8 and L3. (B) Bone marrow aspiration demonstrated active hyperplasia and a blast cell count of $90.0 \%$.

physical examination, pallor was observed most prominently at the face, eyelids, lips and palms. No lymphadenopathy or hepatosplenomegaly were identified. The findings of a neurologic examination, including strength testing, were normal, and other examinations were unremarkable.

A complete blood count revealed the following: hemoglobin level, $9.3 \mathrm{~g} / \mathrm{dl}$; white blood cell count, 4,780/ $\mu \mathrm{l}(\mathrm{NR}$, $3,500-9,500 / \mu 1$ ) with $3 \%$ blast cells; platelet count, $21,000 / \mu 1$ (NR, 100,000-300,000/ $\mu 1$ ); lactate dehydrogenase, 1,082 IU/1 (NR, 72-182 IU/l); alkaline phosphatase, $6.72 \mu \mathrm{g} / 1$ (NR, 11.4-24.6 $\mu \mathrm{g} / 1$ ); Type I collagen carboxyl terminal peptide, $0.125 \mathrm{ng} / \mathrm{ml}$ (NR, 0.3-0.58 ng/ml); calcium level, $2.16 \mathrm{mmol} / 1$ (NR, 2.1-2.7 mmol/1); and inorganic phosphorus level, $0.74 \mathrm{mmol} / \mathrm{l}$ (NR, 0.81-1.45 mmol/l). Magnetic resonance imaging (MRI) scans revealed an impaired physiological curve of thoracolumbar spine, clusters of iso/hypo mixed signals and pathological fracture in T8 and L3 with inhomogeneous contrast enhancement (Fig. 1A). A chest CT image demonstrated bone destruction of the sternum, thoracic vertebra, bilateral rib and scapular. In addition, an X-ray showed sheet and irregular low-density lesions on the cranial and maxillofacial bone, pelvis and bilateral proximal femur. Based on this data, a diagnosis of multiple myeloma (MM) was initially made. However, immunofixation electrophoresis (IFE), serum protein electrophoresis (SPE) and urine light chain detection indicated no evidence of monoclonal protein. Furthermore, a bone marrow smear identified active hyperplasia and a blast cell count of $90.0 \%$ (Fig. 1B), while the patient was peroxidase-negative. The immunophenotype of the leukemic blasts was analyzed by flow cytometry (FCM) and was as high as the typical phenotype of common-B-cell ALL patients, with $\mathrm{CD}_{10}{ }^{+}, \mathrm{CD}^{+} 9^{+}$, $\mathrm{CD}_{13}{ }^{+}, \mathrm{CD} 20^{+}, \mathrm{cCD} 7 \mathrm{a}^{+}, \mathrm{CD}_{3}{ }^{+}$and HLA-DR ${ }^{+}$. Cytogenetic analysis revealed 46,XY,t(9;22)(q34;q11) [20/20], while a molecular biology examination demonstrated that the patient was $B C R / A B L$-positive, as determined by a reverse transcription-polymerase chain reaction method (5).
Therefore, a diagnosis of $\mathrm{Ph}^{+}$ALL was ultimately determined according to the Morphology, Immunology, Cytogenetics, Molecular Biology criteria (6).

In addition to administration of $90 \mathrm{mg}$ pamidronate disodium (Bonin; Shenzhen Neptunus Bioengineering Co., Ltd., Shenzhen, China) to reduce bone pain, the patient was treated with the IVD regimen (imatinib, $400 \mathrm{mg} /$ day; vindesine, $4 \mathrm{mg} / \mathrm{day}$, on days 1, 8, 15 and 22; dexamethasone, $10 \mathrm{mg} / \mathrm{m}^{2} /$ day, on days $1-5,8-12,15-19$ and 22-26). Hematological $\mathrm{CR}$ with rapid resolution of pain was observed 2 weeks after the termination of inductive treatment, which was confirmed by a bone marrow smear and FCM. Meanwhile, the patient continued extramural treatment with imatinib at $400 \mathrm{mg} /$ day. Due to the lack of a matched donor, bone marrow transplantation was not considered for this case. During subsequent consolidation therapy, the CAM (cyclophosphamide, $750 \mathrm{mg} / \mathrm{m}^{2}$, days 1 and 8; cytarabine, $100 \mathrm{mg} / \mathrm{m}^{2}$, days $1-3$ and 8-10; 6-mercaptopurine, $60 \mathrm{mg} / \mathrm{m}^{2}$, days 1-7), ML (methotrexate, $3.0 \mathrm{~g} / \mathrm{m}^{2}$, day 1 ; L-asparaginase, 6,000 IU/m ${ }^{2}$, days 2 and 3) and MA (mitoxantrone, $8.0 \mathrm{~g} / \mathrm{m}^{2}$, days $1-3$; cytarabine, $1.5 \mathrm{~g} / \mathrm{m}^{2}$, days $1-3$ ) chemotherapies were applied for $\sim 1$ month, respectively. Prior to each consolidation regimen, a lumbar puncture combined with an intrathecal injection was performed using $10 \mathrm{mg}$ methotrexate, $30 \mathrm{mg}$ cytarabine and $5 \mathrm{mg}$ dexamethasone. However, following intensive post-remission chemotherapy, the patient experienced bone marrow suppression with a significantly reduced total leukocyte count of $170 / \mu 1$ (normal range, 4,000-10,000/ $\mu 1$ ) and was affected by severe pneumonia. A 3-week course of intravenous antibiotics was administrated and the hemopoietic function of the bone marrow returned to the normal levels 1 month later. Simultaneously, molecular CR was confirmed by bone marrow examination, revealing suppression of $B C R / A B L$ chimeric gene expression. Subsequently, the patient was subjected to consolidation treatment with the IVD regimen (imatinib, $400 \mathrm{mg} / \mathrm{day}$; vindesine, $4 \mathrm{mg} / \mathrm{day}$, days 1 and 
11; dexamethasone, $10 \mathrm{mg} / \mathrm{m}^{2} /$ day, days $1-5$ and days $11-15$ ) combined with interferon- $\alpha 2 b$ (3 million units, twice per week) to maintain remission for 9 courses on a periodic basis (every 1 month).

Until November 2013, the patient remained asymptomatic, while sustained hematological and molecular genetic normality were achieved for $\sim 16$ months after CR. In addition, a chest CT image revealed that the bone cortex margin of the left rib was irregular and bone density was uneven in osseous thorax without evident osteolytic destruction. However, in November 2013, the patient was lost to follow-up.

The present was approved by the ethics committees of West China Hospital, Sichuan University (Chengdu, China) and written informed consent was obtained from the patient.

\section{Discussion}

Symptoms of ALL at diagnosis usually include nonspecific manifestation of bone marrow failure, such as fever, weakness or bleeding (7). Although the presence of osteopenia/osteoporosis may be observed in both the initial and progressive phases of patients with ALL, bone pain and spontaneous bone fractures, which mainly involve the proximal limbs due to leukemic involvement along with adjacent joint pain or swelling, as the sole prodrome for ALL are rarely observed (8). As previously described, vertebral body compression fractures associated with bone pain occurred in $<10 \%$ of children with ALL as the initial presentation (9). However, to the best of our knowledge, generalized osteopenia and vertebral complications presented as the only symptoms prior to the diagnosis of ALL has not been previously reported in an adult ALL patient. Based on a search of the PubMed database, to the best of our knowledge only 3 adolescent and 1 child cases have been described in the English literature to date (9-12). These cases presented with exclusive back pain and were then verified to exhibit marked osteoporosis and spontaneous vertebral compression fractures; ultimately, they were diagnosed with ALL after 3-4 months. These cases included an 8-year-old girl in Italy (10) and a 13-year-old boy in Turkey (9), as well as a 9-year-old boy and a 2-year-old boy in Bangladesh $(11,12)$. Compression fractures of the vertebrae are known to be caused by osteoporosis (which is the most common cause), trauma to the back and tumors that develop in the bone or spread to the bone from other sites (13). A tumor that develops in the spine, including MM, is more common in older people (14). In the 56-year-old patient of the present study, a working diagnosis of MM was preliminarily established; thereby, IFE and SPE were applied to exclude MM. Notably, bone destruction is not currently the determined predictor for ALL prognosis; however, bone pain and fractures may still be major evidence to support a diagnosis of ALL and greatly influence the quality of life prior to establishing the ultimate diagnosis of ALL (15). Thus, ALL must be considered in the differential diagnosis, and close follow-up and bone marrow examination are important when a patient presents with unexplained marked osteopenia, bone pain and multiple fractures, particularly prior to establishing a definite diagnosis of idiopathic juvenile osteoporosis for children and MM for adults. In order to suppress bone resorption and reduce bone turnover, the case was treated with pamidronate disodium with no significant side-effects in the entire therapeutic course.
However, the role of imatinib in $\mathrm{Ph}^{+} \mathrm{ALL}$ treatment has attracted increased attention due to the success of TKI treatment of chronic myeloid leukemia. Prior to the discovery of TKIs, $\mathrm{Ph}^{+}$ALL was considered to be the high-risk group with the poorest outcome among all subtypes of ALL (16). Although a CR rate of $50-60 \%$ could be achieved by routine chemotherapy, short-term remission and high relapse rate resulted in a poor 5-year survival rate of $<10 \%$ for adults and OS of only $20 \%$ (17). More intensive chemotherapy regimens were only able to improve the CR rate, rather than the long-term OS. However, with the use of TKIs in chemotherapy, the therapeutic efficacy markedly increased, reaching up to $95 \%$ in patients undergoing CR and $70 \%$ in those undergoing molecular CR, as well as resulted in improved 3 -year OS of 55\% $(18,19)$. For older patients (age, $\geq 65$ years) with $\mathrm{Ph}^{+} \mathrm{ALL}$, imatinib alone or in combination with reduced intensive chemotherapy is reported to be the first-line inductive regimen based on the National Comprehensive Cancer Network Guidelines since 2012 (4). In addition, the final results of the EWALL-Ph-01 Study were presented in 2012 at the American Society of Hematology meeting and recommended the used of the third generation TKI, dasatinib, and low intensity chemotherapy as the first-line treatment in patients with de novo $\mathrm{Ph}^{+}$ALL aged $\geq 55$ years $(20)$.

The 56-year-old male patient of the present study successfully completed the IVD regimen and quickly achieved CR 2 weeks after initiation of the treatment. Whether optimal long-term efficacy may be maintained using an imatinib-based regimen, allogeneic hematopoietic stem cell transplantation (Allo-HSCT) or TKI + Allo-HSCT requires further investigation. Certain authors have recommended that TKI-based regimens may have a similar or even superior effect compared with Allo-HSCT, particularly in patients who may be at a high risk of transplantation-associated mortality, such as the elderly (21). Due to the lack of a matched donor, the current case accepted regular intensification and continued extramural imatinib as a consolidation approach. In addition, clinical studies are currently ongoing to evaluate the best procedure and duration of maintenance in patients who previously benefited from TKI-based front-line treatment. Until recently, maintenance therapy with imatinib or interferon demonstrated impressive effectiveness for the treatment of patients with $\mathrm{Ph}^{+}$ALL (22).

In conclusion, based on the aforementioned evidence, the present case was subjected to interim maintenance treatment using imatinib and IFN to achieve persistent molecular CR for $\sim 16$ months. The emergence of TKIs has greatly contributed to marked improvement in the outcome of patients with $\mathrm{Ph}^{+} \mathrm{ALL}$; thus, there has been much debate regarding whether regimens including TKI could challenge the efficacy of conventional high-dose chemotherapy for such cases. To a certain extent, the present study suggests that the administration of TKIs may provide certain additional benefits for patients with $\mathrm{Ph}^{+} \mathrm{ALL}$ compared with high-dose chemotherapy. For example, a common side effect of high-dose chemotherapy is poor tolerance, however, TKIs appear to be associated with improved tolerance. Further evidence and studies from additional multicenter, prospective, randomized clinical trials are required to clarify the role of reduced-intensity chemotherapy combined with imatinib for the treatment of adults with $\mathrm{Ph}^{+} \mathrm{ALL}$. 


\section{Acknowledgements}

The authors would like to thank the patients and the staff who participated in the present study. This study was funded by a grant from the National Natural Science Foundation of China for Young Scholars (no. 81302148).

\section{References}

1. Hunger SP and Mullighan CG: Redefining ALL classification: Toward detecting high-risk ALL and implementing precision medicine. Blood 125: 3977-3987, 2015.

2. Te Winkel ML, Peiters R, Wind EJ, Bessems JH and van den Heuvel-Eibrink MM: Management and treatment of osteonecrosis in children and adolescents with acute lymphoblastic leukemia. Haematologica 99: 430-436, 2014.

3. Nair M, Kusumakumary P and Nair PS: Rare presentation of pediatric acute promyelocytic leukemia as multiple lytic bone lesions: Case report and review of the literature. J Cancer Res Ther 10: 381-383, 2014.

4. Chalandon Y, Thomas X, Hayette S, Cayuela JM, Abbal C, Huguet F, Raffoux E, Leguay T, Rousselot P, Lepretre S, et al; Group for Research on Adult Acute Lymphoblastic Leukemia (GRAALL): Randomized study of reduced-intensity chemotherapy combined with imatinib in adults with $\mathrm{Ph}$-positive acute lymphoblastic leukemia. Blood 125: 3711-3719, 2015.

5. Ross DM, Branford S, Moore S and Hughes TP: Limited clinical value of regular bone marrow cytogenetic analysis in imatinib-treated chronic phase CML patients monitored by RQ-PCR for BCR-ABL. Leukemia 20: 664-670, 2006.

6. Matutes E, Pickl WF, Van't Veer M, Morilla R, Swansbury J, Strobl H, Attarbaschi A, Hopfinger G, Ashley S, Bene MC, et al: Mixed-phenotype acute leukemia: Clinical and laboratory features and outcome in 100 patients defined according to the WHO 2008 classification. Blood 117: 3163-3171, 2011.

7. Faderl S, O'Brien S, Pui CH, Stock W, Wetzler M, Hoelzer D and Kantarjian HM: Adult acute lymphoblastic leukemia: Concepts and strategies. Cancer 116: 1165-1176, 2010.

8. Leblicq C, Laverdière C, Decarie JC, Delisle JF, Isler MH, Moghrabi A, Chabot G and Alos N: Effectiveness of pamidronate as treatment of symptomatic osteonecrosis occurring in children treated for acute lymphoblastic leukemia. Pediatr Blood Cancer 60: 741-747, 2013

9. Atas A, Cakmak A, Soran M, Soker M and Varma M: Severe osteoporosis and high level TSH in a child before the diagnosis of acute lymphoblastic leukemia. J Pediat Hematol Oncol 31: 588-591, 2009.

10. Bertuna G, Famà P, Lo Nigro L, Russo-Mancuso G and Di Cataldo A: Marked osteoporosis and spontaneous vertebral fractures in children: Don't forget, it could be leukemia. Med Pediatr Oncol 41: 450-451, 2003.
11. Hafiz MG and Islam A: Extensive skeletal lesions in childhood acute lymphoblastic leukemia. Mymensingh Med J 18: 88-94, 2009.

12. Hafiz MG, Islam A and Siddique R: Back pain and vertebral compression: An unusual presentation of childhood acute lymphoblastic leukemia. Mymensingh Med J 19: 130-136, 2010.

13. Kim DH and Vaccaro AR: Osteoporotic compression fractures of the spine; current options and considerations for treatment. Spine J 6: 479-487, 2006.

14. Kaloostian PE, Zadnik PL, Etame AB, Vrionis FD, Gokaslan ZL and Sciubba DM: Surgical management of primary and metastatic spinal tumors. Cancer Control 21: 133-139, 2014.

15. Shah NR, Landi DB, Kreissman SG, Kulbachi E and Moran C: Presentation and outcomes for children with bone marrow necrosis and acute lymphoblastic leukemia: A literature review. J Pediatr Hematol Oncol 33: e316-e319, 2011.

16. Usuki K, Tojo A, Maeda Y, Kobayashi Y, Matsuda A, Ohyashiki K, Nakaseko C, Kawaguchi T, Tanaka H, Miyamura K, et al: Efficacy and safety of nilotinib in Japanese patients with imatinib-resistant or -intolerant $\mathrm{Ph}+\mathrm{CML}$ or relapsed/refractory Ph+ ALL: A 36-month analysis of a phase I and II study. Int J Hematol 95: 409-419, 2012.

17. Thomas DA, Faderl S, Cortes J, O'Brien S, Giles FJ, Kornblau SM, Garcia-Manero G, Keating MJ, Andreeff M, Jeha S, et al: Treatment of Philadelphia chromosome-positive acute lymphocytic leukemia with hyper-CVAD and imatinib mesylate. Blood 103: 4396-4407, 2004.

18. Mizuta S, Matsuo K, Maeda T, Yujiri T, Hatta Y, Kimura Y, Ueda Y, Kanamori H, Usui N, Akiyama $\mathrm{H}$, et al: Prognostic factors influencing clinical outcome of allogeneic hematopoietic stem cell transplantation following imatinib-based therapy in BCR-ABL-positive ALL. Blood Cancer J 2: e72, 2012.

19. Ohno R: Changing paradigm of the treatment of Philadelphia chromosome-positive acute lymphoblastic leukemia. Curr Hematol Malig R 5: 213-221, 2010.

20. Philippe R, Marie M, Françoise H, et al: Dasatinib (Sprycel ${ }^{\circledR}$ ) and Low Intensity Chemotherapy for First-Line Treatment in Patients with De Novo Philadelphia Positive ALL Aged 55 and Over: Final Results of the EWALL-Ph-01 Study. Blood (ASH Annual Meeting Abstracts) 120: 612, 2012.

21. Mizuta S, Matsuo K, Yagasaki F, et al: Pre-transplant imatinib-based therapy improves the outcome of allogeneic hematopoietic stem cell transplantation for BCR-ABL-positive acute lymphoblastic leukemia. Leukemia 25: 41-47, 2011.

22. Heike P, Sylvia W, A Binckebanck, et al: Imatinib (IM) and interferon-alpha (IFN- $\alpha$ ) maintenance therapy is associated with long-term DFS in a subset of elderly patients with Philadelphia-positive acute lymphoblastic leukemia $\left(\mathrm{Ph}^{+} \mathrm{ALL}\right)$. Blood (ASH Annual Meeting Abstracts) 120: 1503, 2012. 